\title{
Integrated Centralized Substation Protection
}

\author{
A. P. Meliopoulos \\ George Cokkinides \\ Hussain F Albinali \\ Paul Myrda \\ E. Farantatos \\ Fellow, IEEE \\ Member, IEEE \\ Student Member, IEEE \\ Senior Member, IEEE \\ Member, IEEE
}

\begin{abstract}
Substation cyber assets are mission critical for protection and control of substations. Managing and ensuring their secure operation is of paramount importance. A known vulnerability is hidden failures which are responsible for about $10 \%$ of misoperations and their detrimental effects on system reliability. The paper presents an integrated centralized substation protection approach that is based on the recently developed setting-less relays which are integrated into a centralized substation protection scheme with the following features: (a) fast, dependable and secure protection of each substation protection zone by a settingless relay, $(b)$ supervision of each settingless relay by validating relay input data by a substation wide state estimator, (c) self-healing against hidden failures by detecting and identifying compromised data and replacing them with estimated values, thus ensuring that the settingless relays will always operate on validated data. The paper provides a summary review of the settingless protective relay and introduces the Integrated Centralized Substation Protection Scheme (ICSP) which uses the data from all settingless relays in the substation to perform a substation wide state estimation. The state estimator uses a hypothesis testing algorithm to determine whether (a) data are valid with no faults or hidden failures, (b) data are valid and a fault exists in the system, or (c) some data are invalid due to hidden failures. In the last case, the state estimator uses the substation state and model to replace the compromised data with estimated values and thus enabling self-immunization against hidden failures. A byproduct of the method is the substation state estimate which is transmitted to the control center where it is used with the state from all substations to synthesize the system wide state estimate and model. Architectural issues are addressed as well as migration issues of existing systems into the proposed ICSP.
\end{abstract}

Key Words - setting-less relay, centralized substation protection, dynamic state estimation, hidden failures

Glossary: MU: Merging Unit, LN: Logical Node, ICSP: Integrated Centralized Substation Protection

\section{Introduction}

The technological advancements in communications and computational capabilities have paved the way for new approaches in power system protection and control. New approaches are necessary to confront the challenges introduced by the new, revolutionary changes in power systems. These challenges are concurrent with tremendous efforts by utilities to boost system reliability. Such efforts are undermined by mis-operations of the protection system. The rate of relay mis-operation, as a percentage of total operations is approximately 10 percent, as reported by North American Electric Reliability Corporation (NERC) [1]. An analysis of protection failures reveals that the majority occurs because of hidden failures. Such failures are defined in [2] as "permanent defect[s] that will cause a relay or a relay system to incorrectly and inappropriately remove a circuit element(s) as a direct consequence of another switching event."

Present numerical relays have a limited capability to detect hidden failures. Presently, relays are not capable to correct the effects of the hidden failures nor they have the capability to detect the true condition of the power system in the presence of hidden failures. As a result, when a hidden failure is detected the only option is to inhibit the protection function. The affected protection system depends on other protection systems, unaffected by the hidden failure(s), to take the proper action. It should be apparent the protection system reliability is decreased.

Relay manufacturers and researchers have proposed approaches that could minimize the effects of hidden failures and cope with other complexities. Along these lines, adaptive protection schemes and voting schemes have been proposed. Adaptive protection schemes have been proven to be complex and do not easily meet the speed that is required for protection. Voting schemes are not reliable as a hidden failure may affect several relays that participate in the voting - in this case the majority of relays may be taking a wrong decision.

In response to these challenges, several new concepts have emerged. The idea of setting-less protection was inspired by the differential protection function, which is simple, features minimal settings, and most 
importantly, does not require coordination with other protective relay functions [3], [4]. The setting-less protection relay monitors all physical laws that the device under protection must satisfy when it operates normally (i.e. unfaulted). This monitoring is accomplished by performing dynamic state estimation (DSE) using sampled value measurements. DSE continuously compares the collected measurements (i.e., sampled values) to the dynamic model of the protection zone and provides a quantitative assessment of how well the data fit the dynamic model. Specifically, the chi-square test calculates the probability that the measurements fit the model. The chi-square test typically returns a probability of $100 \%$ for healthy protection zones and $0 \%$ for protection zones with any type of fault. Setting-less relays, as any other type of relay, are vulnerable to hidden failures which corrupt the measured values. Hence, a mechanism to detect such failures is essential for reliable protection of power systems.

The objective of this paper is to incorporate settingless relays into an integrated centralized substation protection scheme (i.e., Integrated Centralized Substation Protection (ICSP)). The ICSP supervises the validity of the measured values streaming into the setting-less relays by performing a substation-wide state estimation, using data from all setting-less relays in the substation. In case that compromised data are detected, the ICSP has the capability to replace the compromised data with corrected data using the state estimates and the real time model. The paper details the computational procedures of the ICSP as well as the proposed system architecture. Section II presents the ICSP in as much detail as practical, given paper length restrictions. Section III discusses the architecture of the ICSP. Finally, Section IV provides an illustrative example. Section $\mathrm{V}$ provides concluding remarks and section VI discusses the usefulness of the proposed ICSP in providing the basic infrastructure for the energy management system of the future.

\section{Integrated Centralized Substation Protection}

We present an approach for reliable monitoring of the overall protection system. We prefer an implementation using merging units and setting-less relays for protection zones. The overall proposed structure is shown in Figure 1. The system consists of the instrumentation, the merging units, the settingless relays that provide protection for each protection zone, communications channels between relays and controls and telemetering and a substation wide quasi-dynamic state estimation to continuously monitor the integrity of the overall system. In our implementation, at the setting-less relays, the dynamic state estimation is performed 40 times per cycle using sampled values obtained with the merging units. This accommodates the standard sampling rate of 80 samples per cycle. This process has been demonstrated with extensive numerical experiments, in the laboratory and in the field.

The setting-less relay can also compute the phasors of the $\mathrm{V}$ and I measurements and stream the phasor data to the station bus as indicated in Figures 1 and 2. The phasor measurements are used to perform a substation-wide state estimator. All the measurements from all relays represent a high data redundancy for the substation wide state estimator. We performed an analysis of actual substations and the redundancy is above $2000 \%$ meaning that the number of measurements from all relays is typically 20 times the number of state variables describing the substation state. The substation-wide state estimator is implemented with three additional important applications: (a) detection of bad data, (b) hypothesis testing, and (c) correction/replacement of compromised data, if any. The large redundancy makes these applications robust and provides the mechanism by which the individual setting-less relays are supervised and ensure that they will always operate with validated data. A concise description of these functions is provided next.

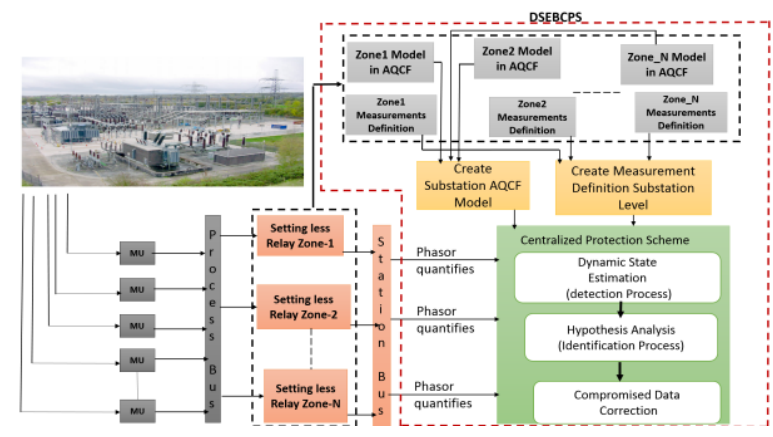

Figure 1: Illustration of Overall Approach

Substation-Wide Dynamic State Estimation Module The substation-wide dynamic state estimation module operates on the entire measurement set of the substation. No other information is needed. The DSE computes the best estimate of the substation dynamic state. Subsequently, the goodness of fit between the measurements and the substation model is computed via the well-known chi-square test which calculates the probability of goodness of fit between the measurement and the substation model. This probability is also referred to as confidence level. A high probability of goodness of fit, i.e. more than 0.90 results in declaring all the data in the substation 
valid. In this case no further action is required. Otherwise, the hypothesis module is called to identify the root cause of the bad data via hypothesis testing and correct/replace compromised data.

Hypothesis Testing Module. This module is initiated when the dynamic state estimation has declared the existence of bad data (data abnormality). The objective of this module is to identify the root cause of the abnormality, i.e. power fault or hidden failure or both. There exist three possibilities: (a) one or more power faults exist in the system, (b) one or more hidden failures, or (c) simultaneous occurrence of faults and hidden failures. The probability of having two simultaneous faults or two simultaneous hidden failures within the substation is very low. Realistically, we consider the following possible events as most likely to occur: (a) occurrence of a hidden failure, (b) occurrence of a power fault, and (c) simultaneous occurrence of a power fault and a hidden failure. Other possible events will have insignificant probabilities of occurring simultaneously.

The hypothesis testing is guided by the following observations: (a) at the substation level the redundancy is high (over 2000\%) - this implies that the existence of leveraging points are not existent and therefore normalized residuals from the state estimator can be used as guidance of where the problem is located. (b) System is continuously running - this implies that any abnormal event will be captured in real time. The probability of simultaneous failure events occurring at exactly the same time is low. Thus the hypotheses to be considered should be limited to first order events.

The mechanics of hypothesis testing are as follows: First, suspect measurements are identified from normalized residuals which are provided by the DSE. Second, suspect measurements are grouped using the source of the data (merging units) and also by protection zone. Third, protection zones are identified for which the protection functions have reported a fault. Subsequently, three types of hypothesis testing are performed as follows:

Hypothesis Type 1 (H1): Remove suspect measurements and rerun DSE. If probability is high (more than 0.9), then: (a) removed measurements are bad, (b) identify root cause, (c) issue diagnostics, and (d) replace bad data with estimated values. End hypothesis testing. Otherwise go to $\mathrm{H} 2$.

Hypothesis Type 2 (H2): (determine if a fault decision is correct). For the reported faulted device, remove all internal device measurements and remove the faulted device model from the substation model. Then rerun DSE. If probability is high (more than
0.9), then: (a) the device/protection zone is truly experiencing an internal fault. Allow zone settingless relay to trip the faulted device. End hypothesis testing.

Hypothesis Type 3 (H3): This test combines type 1 and type 2 hypothesis testing to cover the case of a simultaneous fault and a hidden failure.

Data Correction Module. The data correction/replacement module is executed when a hidden failure of instrumentation channels has been identified. The hypothesis testing has identified a number of compromised measurements. Then using the model of the entire substation and the real time operating conditions (both provided by the DSE) the physical quantities represented by these measurements are computed (estimated values). The computed quantities are sampled values. Subsequently, the ICSP streams the estimated values of the compromised data into the process bus, replacing the actual data collected by the merging units. Now any computing device, including the setting-less relays, using the sampled values at the process bus will be using corrected data.

To complete the picture, the substation-wide state estimator also communicates with the control center (EMS). Specifically, the validated results (substation state estimate) from the state estimator are streamed to the EMS where the results from all substations can be time aligned and synthesize the operating conditions (state) of the entire system. It is emphasized that the proposed approach facilitates efficient communications. Specifically, each substation sends to the EMS only the substation state which comprises a very small number of data. When connectivity changes, then connectivity data are transmitted by exception. Similarly if model changes occur, the new mathematical model will be transmitted by exception. The end result is that while the merging units in a substation may be collecting data at rates of millions of data points per second (sampled values), the frequency domain state (phasors) are only a few tens of data points per cycle and per substation.

In summary, the ICSP provides a real time system for supervising the operation of the setting-less relays in a substation and ensuring that the data streaming into these relays are validated data. More importantly, in case of hidden failures, the data compromised by the hidden failure are replaced with estimated values form the validated substation model and operating state to ensure the continuous dependable and secure operation of the relays. The ICSP provides selfhealing capability for the system in case of hidden failures. The proposed ICSP represents a new approach which has become possible with technology that has been developed only in the past few years. 


\section{Communications and Data Management}

Critical to this work is the ability to manage data and communications between the various devices (i.e., setting-less relays and ICSP) within the substation and also between the substation and the control center. Therefore, we have defined the details of the system architecture, which specifies data management, communication protocols, and the hierarchical structure of the system. Figure 2 illustrates the overall proposed architecture with emphasis on the data flow and data handling. The lower part of the Figure shows the architecture to deal with sampled values. Note that all SV are concentrated on four circular buffers which are handled with the sample value data concentrator (SVDC). The four circular buffers handle the local SVs and the telemetered SV with full redundancy (one primary for the local SV and one backup as well as one primary for the telemetered SV and one backup). The SVDC time aligns the SVs as they may be coming from the various merging units with different time latencies and orders them in the circular buffer. Time synchronization is critical for reliable operation of both setting-less relays and centralized protection schemes. The required timing accuracy for such applications is less than $1 \mu \mathrm{s}$. Accordingly, we propose using the IEEE 1588v2 time synchronization standard known as the precision time protocol (PTP) as illustrated in Figure 2. This protocol operates through a data network and can achieve time accuracy of less than $1 \mu$ s.

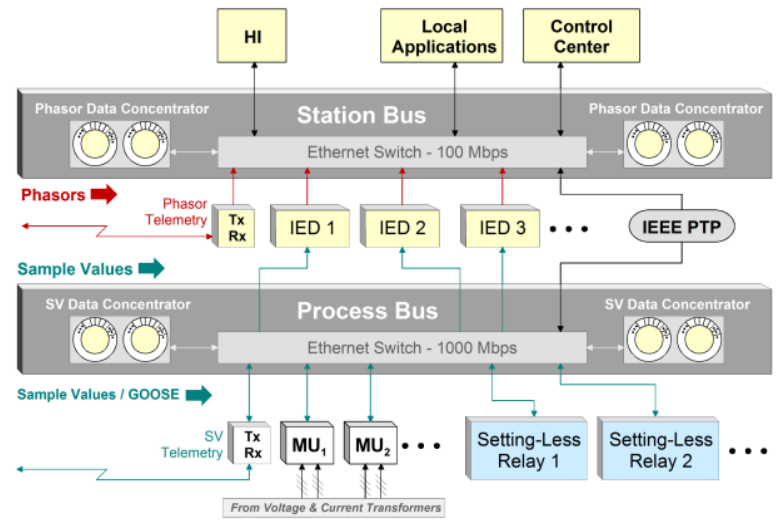

Figure 2: Overall Architecture of the Proposed Scheme

The upper part of the Figure shows a similar architecture for phasor data, indicated as station bus. The station bus facilitates data exchange between setting-less relays and ICSP. The scheme also accommodates telemetered phasor data. This includes phasor quantities streaming from setting-less relays to ICSP as well as telemetered phasors at a rate of up to 1 sample/cycle. Moreover, upon detecting hidden failure the ICSP uses the estimated state and the real time model of the substation to estimate the sampled values that are affected by the hidden failure and streams the estimated SV to the process bus replacing the compromised data. This way the setting-less relays will be now operating on validated data.

The figure also shows communication links to other substations and the control center. The availability of the data on circular buffers facilitates accessibility of the data from any application.

Control functions, not shown in the figure, are executed with GOOSE messaging. GOOSE message exchanges between the IED and ICSP takes place in the station bus. These GOOSE messages include but are not limited to breaker status, disconnect status, setting-less relays operation status, and ICSP permissive signal to setting-less relays.

\section{Illustrative Example}

The concept of ICSP has been tested with numerous numerical experiments. Here we present results/numerical experiment on a relatively small substation which comprises five protection zones, as shown in Figures 3 and 4 [5]. Specifically, we consider only the following five Protection Zones of the substation: (a) $115 \mathrm{kV}$ Transmission Line, (b) 115 kV Bus, (c) 115/13.8 kV, 36 MVA Transformer, (d) $13.8 \mathrm{kV}$ Bus, and (e) $13.8 \mathrm{kV}$ Distribution Line (one of the two). The objective of the numerical experiments was to prove the concept and show that the system can meet the time response requirements of a protection system so that can be implemented on a larger scale.

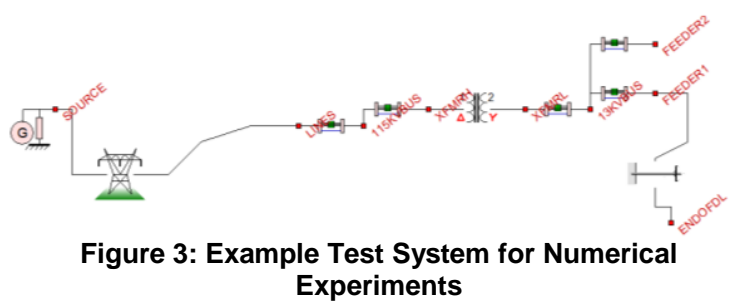

We present a simulated a hidden failure scenario of CT reverse polarity. Typically, this event occurs following pre-commissioning activities for a new substation or maintenance activities for an existing substation. In either case, the problem may remain hidden following the energization until the effected zone is loaded. This case simulates the CT reverse polarity to analyze the response of both the settingless relay and the ICSP. Specifically, we modeled CT10 (see figure 4) phases A, B, and C, which provide the setting-less relay of the transformer zone with the current measurements at the transformer secondary, with reverse polarity by swapping the terminals of 
each CT at the relay terminals. The sequence of events starts with energizing the substation without load. Then, the transformer is loaded with a $15 \mathrm{MW}$ load at time $\mathrm{t}=3 \mathrm{~s}$.

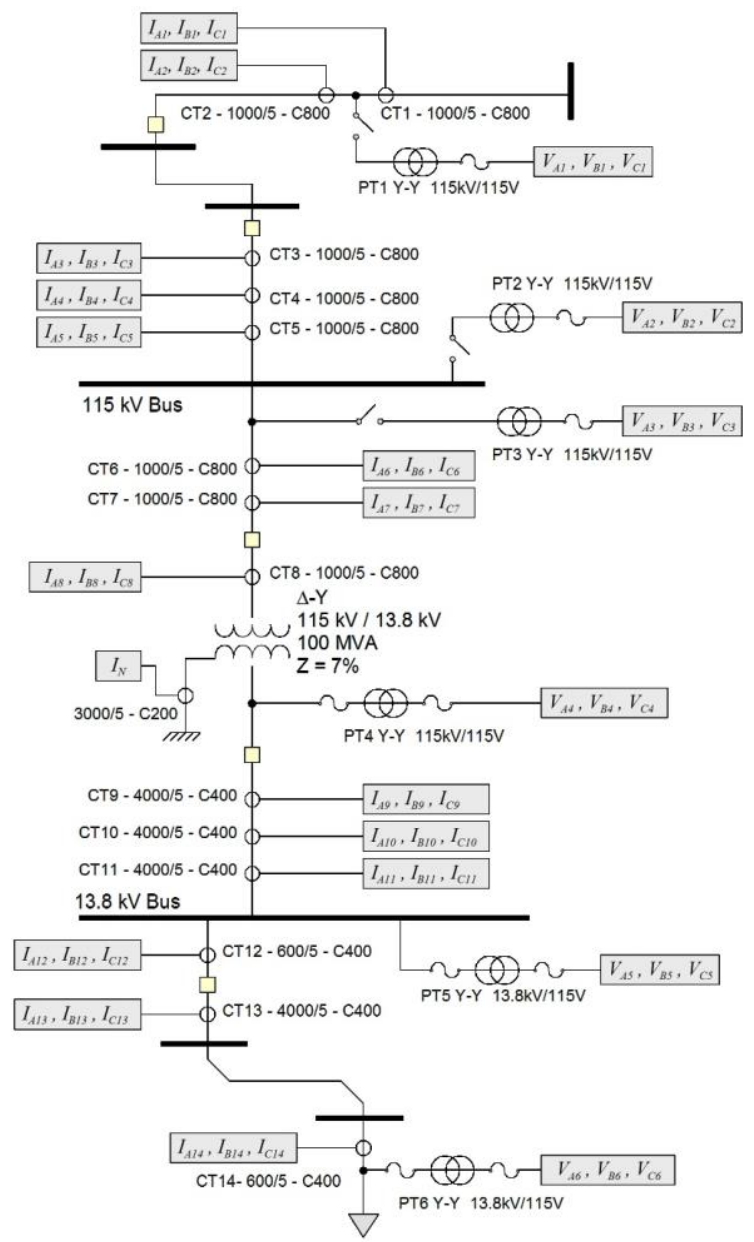

Figure 4: Example Test System: One line diagram with instrumentation

Figure 5 shows the electric current waveforms of the secondary side of the transformer (CT-9 and CT10) depicted from the setting-less relay of the transformer zone. The figure shows a 180 degreephase shift between the current waveforms extracted from CT 9 (i.e., the healthy CT) and CT 10 because of the reverse polarity. Figure 5 also depicts the settingless relay operations for this event, which show that the relay operated and initiated a trip signal to isolate the transformer. This response is a relay mis-operation because of the hidden failure.

The ICSP responded to the event of the hidden failure by fisrt detecting bad data via the hypothesis testing and then initiating hypothesis testing. The ICSP computed normalized residuals show high values. Figure 6 shows the nine highest values of the normalized residuals. Note that the measurement from CT-10, phase B, has the highest normalized residual. The common mode criteria verification revealed that all three phases measurements extracted from CT-10 exceeded the threshold. The threshold is a user selected parameter; in this case we used the value of 2 . Consequently, the measurements extracted from CT10 are classified as suspicious measurements. The ICSP initiated hypothesis testing by removing the CT10 measurements. The hypothesis testing was successful and returned a high confidence level. The ICSP concluded that there is a hidden failure within the substation and did not detect any faulty zones. Additionally, the ICSP precisely identified the instrumentation channels that have been compromised by the hidden failure. Subsequently, the ICSP created a virtual merging unit that computes the phase $\mathrm{A}, \mathrm{B}$ and $C$ currents from the estimated substation state and substation model. The computed estimated sampled values replace the compromised data from CT-10 and attached merging unit. At the same time a diagnostic is issued and transmitted to the control center operator to alarm him/her of the detected hidden failure and the need for a repair. This case demonstrates the advantage of redundancy in the measurements, which makes the hypothesis testing very efficient.

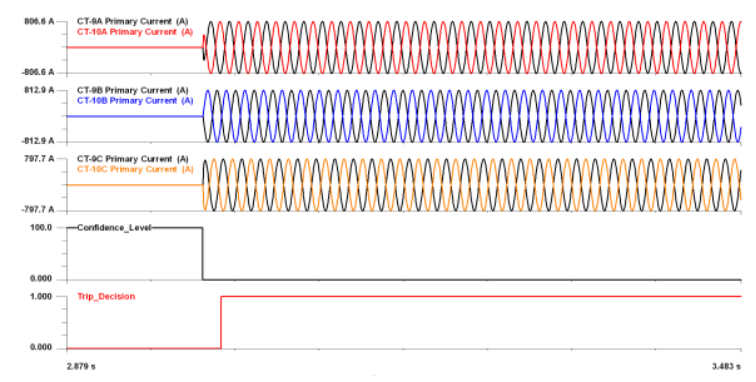

Figure 5: Setting-less relay of the transformer zone for the test case

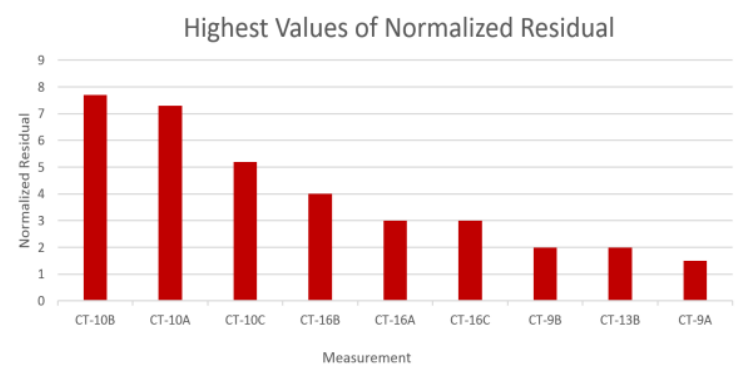

Figure 6: Highest values of the normalized residuals

The detected faulty waveforms in the setting-less relay were replaced with the estimated signals computed in the ICSP and streamed to the setting-less relay, as shown in Figure 7. Note that there is a period of about two cycles after $t=3 \mathrm{~s}$ for which the setting-less relay operates with compromised data from the hidden failure. During this period the setting-less relay reports zero confidence level. At time $t=3.032 \mathrm{~s}$ the corrected data start arriving at the process bus and the settingless relay now operates on validated data. Note on the figure the reversal of the electric current data and the almost immediate response of the setting-less relay to 
$100 \%$ confidence level. This process demonstrates the speed of the ICSP to self-heal the protection system within two cycles and enable the correct operation of the setting-less relay even in the presence of the hidden failure.

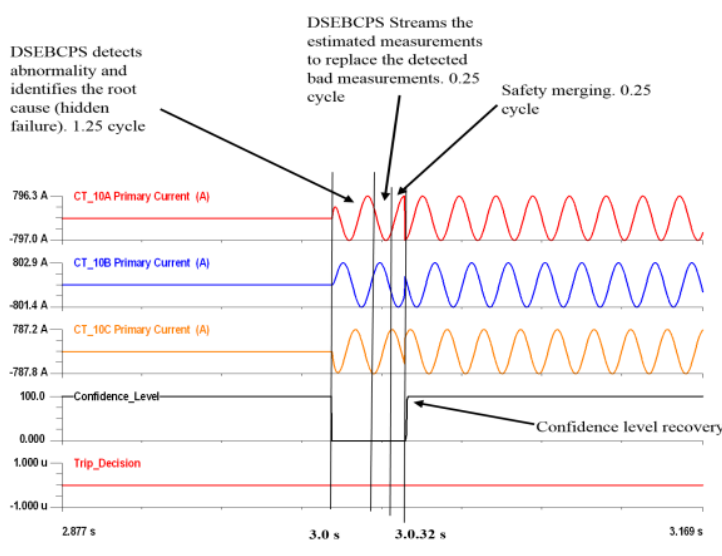

Figure 7: Setting-less relay corrected response

\section{Conclusions}

This paper proposes an Integrated Centralized Substation Protection (ICSP) scheme, which operates at the substation level. It has the capability to detect hidden failures in real time and to take corrective action. Specifically it has the following attributes:

- Supervises protection functions of individual protection zones

- Detects and identifies type and location of Hidden Failures. In this case computes and replaces the compromised data and streams the corrected data into the process bus for secure and dependable operation of the setting-less relays.

- Determines existence of faults and in this case allows zone protective relays to operate

The process starts with data streaming from the setting-less relay to the ICSP as well as telemetered data also streaming into the ICSP. The ICSP processes the data by performing substation-wide Dynamic State Estimation. This process verifies the consistency (or inconsistency) between the measurement and the substation model. Upon detecting inconsistency through the chi-square test, the ICSP performs hypothesis testing to identify the root cause of the inconsistencies, i.e. hidden failures or faults or both. We have demonstrated, through numerical experiments, the scheme's capability of detecting hidden failures and to take corrective action in case of hidden failures.
Moreover, we defined the IEC 61850-based architecture of the ICSP with high degree of redundancy at every layer. The architecture entails separating the process bus and the station bus into two separate local networks. We proposed PRP topology for the process and station buses to satisfy the criterion of $0 \mathrm{~s}$ data recovery. Furthermore, we introduced data concentrators at both the station and process buses. The data concentrators aggregate data from different sources and overcome the challenge of data latency. The proposed architecture is fully redundant.

\section{Future Energy Management System}

The proposed ICSP provides an infrastructure that will be fundamental for the future Energy Management Systems. Specifically it provides a distributed dynamic state estimator that makes available the operating state of the system at speeds not available today, i.e. once per cycle. In addition provides all the data collected at a substation at two different time scales, i.e. sampled values (typically 200 microsecond resolution) and phasor values (typically $16 \mathrm{~ms}$ resolution). This data are provided in redundant circular buffers and are available to any other applications.

This infrastructure is necessary for the future Energy Management Systems. The introduction of new resources in power systems with faster controls present new operational challenges which require faster detection of system conditions and faster responses. Decision-making in the future grid requires innovative approaches that overcome the existing limitations. These approaches include advanced data management, sophisticated analytics capabilities, and massive computational capabilities. Our proposed ICSP and its architecture provide the required infrastructure at the substation level for the future energy management system. There is an enormous amount of measurement data at the substation platform, which are managed by the ICSP, the information (substation state and topology) included in the data is extracted and is streamed to the control center. This represents a drastic reduction of data being communicated to the control center (dramatic reduction in throughput) without losing any information about the substation and at a speed that has not been possible with previous technologies. The availability of the accurate substation models at the control center, updated with frequency of once per cycle, provide opportunities for much faster real time applications. This capability make the control center more effective in decision-making and insures a reliable and cost effective operation and control for the power system network. 
The future EMS will utilize the distributed state estimator of the ICSP, as a source of substation real time operating conditions and substation models to support all required control center applications. Specifically, each substation sends through the ICSP to the EMS its real time model and operating conditions. Any substation topology change, switching, tap changes, breaker operation, etc. it will be communicated to the control center with end to end latency of not exceeding 2 cycles. As a matter of fact while the operating conditions of the substation are streamed continuously (up to 60 times per second), the substation model is communicated by exception when a changed has occurred. This way the throughput is drastically reduced. Along with the operating conditions of the substation the ICSP can send substation alarms that can be used for increased awareness of substation conditions and activate any security measurements to insure reliable operation.

The EMS synthesizes the overall system state from the states of each substation in real time. This information can be used in many applications associated with power system economic operation and control as shown in Figure 8. The synthesis of the entire system state from the substation states is straightforward because each substation state is time tagged with GPS accuracy. Simply stated, the states of all substations with the same time tag are combined to provide the system state at that specific time tag. Note that it is important that the time tags are accurate. The ICSP provides an accuracy of one microsecond which is more than enough to guarantee a reliable process.

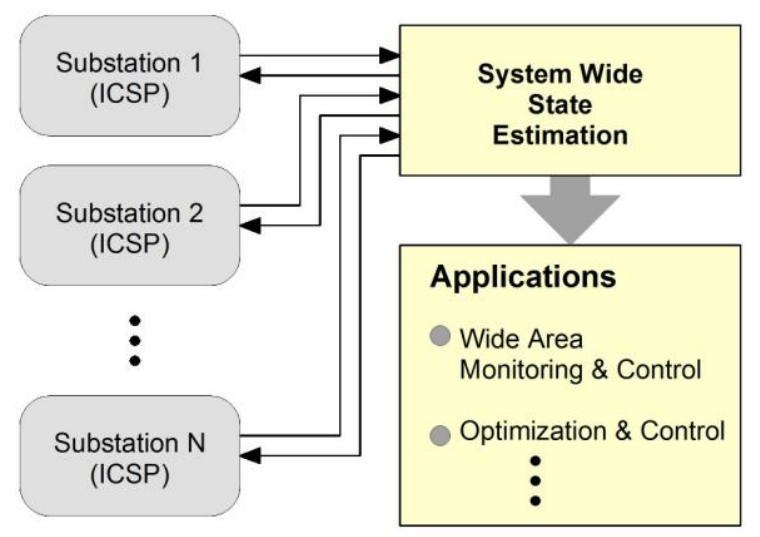

Figure 8: Future Energy Management System

In summary, the ICSP paves the way for advanced (next generation) EMS that is capable to perform several applications with accurate and validated models in real time and overcome the challenges posed by the future power grid.

\section{References}

[1] Protection System Misoperation Task Force, "Misoperations Report," NERC Planning Committee, 2014.

[2] S. Tamronglak, S. H. Horowitz, A. G. Phadke, and J. S. Thorp, "Anatomy of power system blackouts: Preventive relaying strategies," IEEE Transactions on Power Delivery, vol. 11, no. 2, pp. 708-715, Apr. 1996.

[3] S. Meliopoulos et al., "Integration \& automation: From protection to advanced energy management systems," in Bulk Power System Dynamics and Control-IX Optimization, Security and Control of the Emerging Power Grid (IREP), 2013 IREP Symposium, 2013, pp. 1-11.

[4] PSERC Report, "Setting-less protection".

[5] H. Albinali, S. Meliopoulos, "Dynamic State Estimation Based Centralized Protection Scheme" accepeted for PowerTech Confnernce

[6] Kirmann, H., "Seamless redundancy," ABB Report.

[7] McGhee, J., and Goraj, M., "Smart high voltage substation based on IEC 61850 process bus and IEEE 1588 time synchronization," First IEEE International Conference on Smart Grid Communications, Gaithersburg, MD, 2010, pp. 489-494.

[8] Communication networks and systems for power utility automation - Part 9-2: Specific Communication Service Mapping (SCSM) - Sampled values, IEC Standard 61850-9-2, Ed. 2, Mar. 2010.

[9] Retty, H. A., "Evaluation and standardizing of phasor data concentrators," MSc Dissertation, Virginia Polytechnic Institute and State University, 2013.

[10] Power System Relaying Committee, "IEEE Guide for phasor data concentrator requirements for power system protection, control, and monitoring," 2013.

[11] Kanabar, M., Adamiak, M. G., and Rodrigues, J., "Optimizing wide area measurement system architectures with advancements in Phasor Data Concentrators (PDCs), " IEEE Power \& Energy Society General Meeting, Vancouver, BC, 2013, pp. $1-5$. 